Article

\title{
Potential Benefits from Sharing Rainwater Storages Depending on Characteristics in Demand
}

\author{
Yongwon Seo ${ }^{1, *}$, Sun Young Park ${ }^{2, \dagger}$ and Young-Oh Kim ${ }^{3, \dagger}$
}

1 Department of Civil Engineering, Yeungnam University, 280 Daehak-ro, Gyeongsan 712-749, Korea

2 Department of Civil and Environmental Engineering, University of Illinois at Urbana-Champaign, Urbana, IL 61801, USA; E-Mail: spark185@illinois.edu

3 Department of Civil and Environmental Engineering, Seoul National University, 1 Gwanak-ro, Gwanak-gu, Seoul 151-742, Korea; E-Mail: yokim05@snu.ac.kr

$\dagger$ These authors contributed equally to this work.

* Author to whom correspondence should be addressed; E-Mail: yseo@ynu.ac.kr; Tel.: +82-53-810-2883; Fax: +82-53-810-4622.

Academic Editor: Miklas Scholz

Received: 2 December 2014 / Accepted: 28 February 2015 / Published: 11 March 2015

\begin{abstract}
A rainwater harvesting system (RWHS) is an alternative water resource that collects and stores rainwater from rooftops. It helps smooth out variations in water supply and sustainable water resources management. In this study, we investigated the potential benefits from sharing RWHS with nearby neighbors in a community. We introduced different mean, variance, and correlation in water demand for each water user. A simple case of a community composed of four prospective users is introduced to investigate the benefit from sharing RWHSs. Using the historical rainfall records from four catchments in South Korea, a storage-reliability-yield analysis is applied to obtain the required storage and corresponding reliability for a given yield ratio. The results indicate that the required total storage is reduced when RWHSs are shared among users and the rate of reduction is proportional to the degree of sharing. The required total storage was reduced down to $61 \%$ compared to the original storage when RWHSs are shared for a target reliability of $80 \%$. The benefit from sharing also depends on the demands, which are different among users. Test cases with different means and variances as well as correlation disclose the conditions when the benefit from sharing RWHSs is maximized. The result showed that difference in
\end{abstract}


mean and variance additionally reduces the required storage up to $3 \%$ and correlation in demand also as much affects the required storage.

Keywords: rainwater harvesting system; sharing rainwater harvesting system; demand characteristics; reliability; resiliency

\section{Introduction}

Rainwater harvesting is defined as gathering and storage of water running off surfaces on which rain has directly fallen [1]. It could be a potential alternative in small or rural communities that are remote and cannot be served from centralized water supply systems. The benefit of rainwater harvesting can be summarized as follows [2]: First, rainwater harvesting covers peaks in demand that is greater than the flow of the water source. Therefore, it helps smooth out variations in water supply and sustainable water resources management. In addition, rainwater harvesting provides water security in case of supply interruptions or disaster. It has numerous additional benefits in terms of providing grey water for household water use, a source of chemically untreated water, reducing peak flows in urban catchments, thermal storage, freeze protection and so forth. A rainwater harvesting system (RWHS) typically consists of natural landforms and artificial facilities that store rainwater in the hydrologic cycle [3]. Due to the benefits of RWHS, it has been adapted for various purposes worldwide. For example, RWHSs were built for potable use [4-7], household irrigation and other uses [3,8,9], and agricultural irrigation in both urban and agricultural areas $[10,11]$. It has been widely used as a practical approach for sustainable water resources management particularly in urban areas [12].

The studies on RWHS have been primarily focused on sizing. The conceptual behavior of a RWHS in terms of yield after spillage (YAS) and yield before spillage (YBS) was firstly introduced by Jenkins et al. [13]. These concepts have been used to evaluate the performance and determine the optimal size of a RWHS [5,11,14]. As a case study, Villarreal and Dixon [9] showed the benefits from a RWHS in terms of improving water saving efficiency. Ghisi et al. [6] showed that the storage size of a RWHS depends on the characteristics of rainwater demand. Guo and Baetz [15] applied the concept of a storm-water detention basin to an RWHS, which was originally proposed by Howard [16], Loganathan and Delleur [17], and Adams and Papa [18]. They also obtained an analytic solution of optimal sizing assuming one-parameter exponential distribution for rainfall. Basinger et al. [8] used nonparametric stochastic rainfall generator for development of a reliability model. Recent researches are more focusing on the reliability analysis of RWHSs. Hanson et al. [19] considered an RWHS as a reservoir and applied the common Storage-Reliability-Yield (SRY) relationship concept to estimate the appropriate size of a RWHS. SRY relationships are used to determine the reservoir storage capacity for delivery of a specified yield with a given reliability and also used to assess the reliability of an existing reservoir system from a reservoir mass balance equation and historic inflow time series or synthetic inflows from a variety of theoretical streamflow models [20]. Imteaz et al. [7,21] performed a reliability analysis of rainwater thanks in Melbourne using daily water balance model. Rahman et al. [22] also performed reliability analysis and evaluate the benefit from a RWHS in Sydney. 
In previous studies, most researchers considered a RWHS as a reservoir and adopted similar approaches, which had been applied to the reliability analysis of reservoir storages, whereas few have considered a possibility of sharing RWHSs. Therefore, the basic idea of this study is to show the potential benefits from sharing RWHSs by establishing sharing networks among individual systems. An individual user is expected to potentially benefit from a sharing network, where such sharing can be easily accomplished. A RWHS-sharing network can have various possible forms. They can be large storages shared by a community or individually owned storages, which are networked through social programs or practices that encourage sharing private RWHSs communally. Seo et al. [23] proposed a framework to evaluate the sharing network and estimate the required storage size depending on the target reliability. Seo et al. [24] also showed that the benefit from a sharing network of RWHS can be even increased under the climate change condition, where the seasonal variability of rainfall increases. They assumed that individual water demands are independent to each other. However, individual water demands can be strongly correlated with each other because water uses are affected by meteorological and environmental factors [25-27].

In this regard, we extended the original idea of sharing RWHSs [23,24] in this study. This study aims at evaluating the performance of RWHSs when they are shared by users in a community. In previous studies, a normal [28,29] and a lognormal distribution for demand [29,30] were applied to calculate a deficit rate for a specific tank storage. In this regard, this study adopted a probabilistic concept for water demands. Particularly, the differences in demands such as mean, variation, and correlation are introduced to evaluate the performance of a RWHS network depending on various conditions. The reliability in RWHS can be defined in a few different ways [31]. In this study, the extended reliability analysis framework [23] from Hanson et al. [19], in which the size of a RWHS is defined for a unit area is utilized to assess the reliability of shared RWHSs in a community. In terms of rainfall, 30 years of daily rainfall records of four catchments in South Korea are used to obtain the size of RWHSs depending on the target reliability.

\section{Methodology}

This study assumes that water demand is a random variable with a probability distribution. The daily water demand is composed of several water uses such as small daily uses and large intermittent uses [9]. Therefore, this study assumes that daily water demand follows a lognormal distribution. Considering the uncertainty of water demand is critical because water demand is inherently variable, depending on the purpose, timing and amount of usage.

\subsection{Characteristics in Water Demand}

This study introduces the correlation in water demand. Individual water uses can be strongly correlated with each other because they are influenced by local meteorology and environment. In addition, the socio-economic living standards of the water users can be similar locally. For simplicity, a constant correlation is assumed for all users. Let $\mathbf{X}$ be a multivariate lognormal random vector with mean $\boldsymbol{\mu}^{\prime}$ and covariance matrix $\boldsymbol{\Sigma}^{\prime}$.

$$
\mathbf{X} \sim\left(e^{\mathrm{Y}_{1}}, \cdots, e^{\mathrm{Y}_{n}}\right)
$$


where

$$
\mathbf{Y}=\left(\mathrm{Y}_{1}, \cdots, \mathrm{Y}_{n}\right) \sim N(\mu, \Sigma)
$$

with mean and variance as follows:

$$
\begin{gathered}
\boldsymbol{E}[\mathbf{Y}]_{i}=e^{\mu_{i}^{\prime}+\frac{1}{2} \sum_{i i}^{\prime}} \\
\operatorname{Var}[\mathbf{Y}]_{i j}=e^{\mu_{i}^{\prime}+\mu_{i}^{\prime}+\frac{1}{2}\left(\sum_{i i}^{\prime}+\sum_{i i}^{\prime}\right)}\left(e^{\frac{1}{2} \sum_{i j}^{\prime}}-1\right)
\end{gathered}
$$

Any covariance matrix is symmetric and real-valued, which can be decomposed as:

$$
\Sigma=C C^{T}
$$

where $\boldsymbol{C}$ is a lower triangular matrix called the Cholesky factor of $\boldsymbol{\Sigma}$, and and $\boldsymbol{C}^{\mathrm{T}}$ is a transposed matrix of $\boldsymbol{C}$. One obtains the correlated random variables by multiplying the vector of uncorrelated random variables by the Cholesky factor.

$$
\mathbf{X}=\mathbf{X}^{u} \boldsymbol{C}^{T}
$$

where $\mathbf{X}^{\mathrm{u}}$ are uncorrelated random variables with a size of $n$ by $m$ ( $m$ observations of length $\left.n\right), \mathbf{X}$ ( $n$ by $m$ ) is correlated random variables according to $\boldsymbol{\Sigma}$ ( $m$ by $m$ ).

In addition to the correlation in demands, this study also incorporates various mean and variances in demands. In the previous study, Seo et al. [23] considered homogeneous and heterogeneous users: homogeneous users with the same mean and variances and heterogeneous users with different means and variances. In contrast, this study introduces more cases to separately evaluate the effect of heterogeneity in mean and variance of water demands. Table 1 lists the test cases with different mean and variance as well as correlation in demands considered in this study. The values of mean $(\mu)$ and

\begin{tabular}{|c|c|c|c|}
\hline Cases & Correlation $(\rho)$ & $\operatorname{Mean}(\mu)$ & Variance $\left(\sigma^{2}\right)$ \\
\hline Same Mean/Same Variance (SMSV) & \multirow[b]{2}{*}{$0,0.90$} & \multirow[b]{2}{*}{$\mu_{1,2,3,4}=0.5$} & $\sigma_{1,2,3,4}^{2}=0.25$ \\
\hline Same Mean/Different Variances (SMDV) & & & $\begin{array}{c}\sigma_{1}^{2}=0.25, \sigma_{2}^{2}=0.50, \\
\sigma_{3}^{2}=0.75, \sigma^{2}{ }_{4}=1.0\end{array}$ \\
\hline Different Means/Same Variance (DMSV) & $0,0.79$ & \multirow{2}{*}{$\begin{array}{l}\mu_{1}=0.2 \\
\mu_{2}=0.4 \\
\mu_{3}=0.6 \\
\mu_{4}=0.8\end{array}$} & $\sigma_{1,2,3,4}^{2}=0.25$ \\
\hline Different Means/Different Variances (DMDV) & $0,0.90$ & & $\begin{aligned} \sigma^{2}{ }_{1} & =0.25, \sigma_{2}^{2}=0.50, \\
\sigma_{3}^{2} & =0.75, \sigma_{4}^{2}=1.00\end{aligned}$ \\
\hline
\end{tabular}
standard deviation $(\alpha)$ are selected from 0 (no yield ratio) and 1 (maximum yield ratio). The values for correlation $(\rho)$ are determined to be 0 and 0.9 . For the DMSV case, the maximum possible correlation for the generation of demand was 0.79 .

Table 1. Test cases with various demand characteristics considered in this study.

\subsection{Networks for RWHSs}

This study introduces four prospective users in order to take account of the benefit from connecting users and forming a sharing network $[23,24]$. All possible network configurations to connect four users are presented in Table 2. It is assumed that every user in the RWHS-sharing community has equal access to all RWHSs within the network. 
Table 2. Rain water Harvesting System (RWHS) sharing networks with a given number of connections.

No. of
Connections $\left(N_{c}\right)$

\subsection{Test Catchments in South Korea}

Historical daily rainfall data from four test catchments (Figure 1) in South Korea was used for simulation. Rainfall data was obtained for the period 1970-1999 (30 years). Details of these test catchments are given in Table 3. The water demand is given as a normalized yield divided by the mean daily rainfall of each catchment per unit area, $\alpha$ as shown in Equation (8). As mentioned earlier, this study introduces four prospective users who share their individual RHWS through a network as shown in Table 2. This study tests various statistics of the water demands of four users as previously described in Table 1 . In Table $3,1 / \lambda, 1 / \xi, 1 / \zeta$, and $1 / \beta$ are the average rainfall duration, interevent time, volume, and intensity.

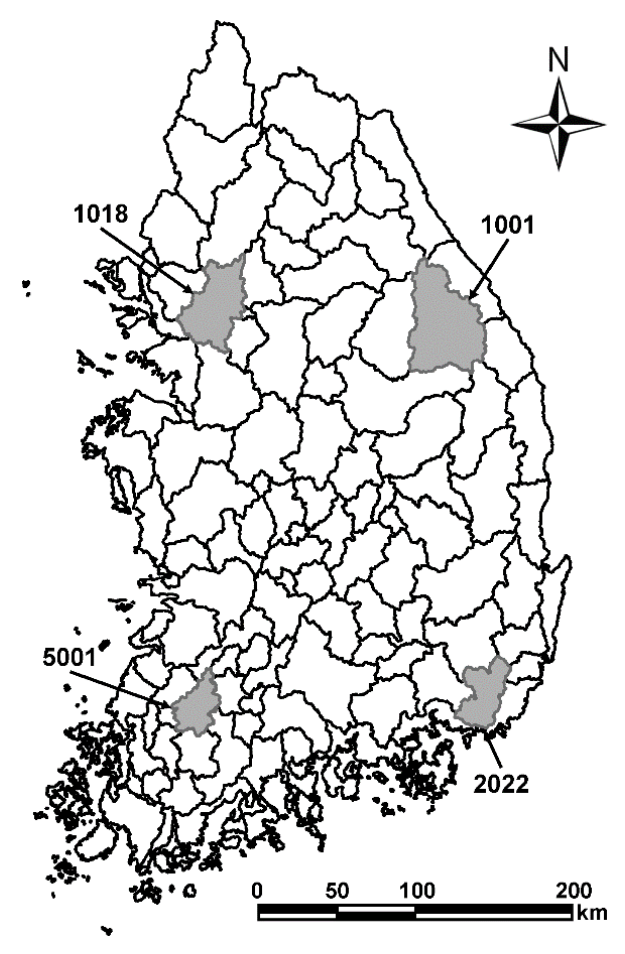

Figure 1. Four test catchments 1001, 1018, 2022, and 5001 in South Korea. 
Table 3. Four test catchments in South Korea.

\begin{tabular}{ccccc}
\hline Catchment & Area $\left.\mathbf{( k m}^{\mathbf{2}}\right)$ & Name of the City & River Name & Yearly Mean Precipitation * $(\mathbf{m m})$ \\
\hline 1001 & 2448 & Gangneung & Han River & 1320 \\
1018 & 1537 & Seoul & Han River & 1248 \\
2022 & 916 & Busan & Nakdong River & 1380 \\
5001 & 715 & Gwangju & Yeongsan River & 1356 \\
\hline
\end{tabular}

Note: * 1970-1999.

\subsection{Storage-Reliability-Yield (SRY) Method}

The required size of a RWHS for a given reliability can be obtained using the SRY curve method [19]. The rainwater storage is given as a function of demand, reliability, and precipitation.

$$
S r=f(X, R, P)
$$

where $S r$ is the total storage volume of RWHSs in the system per unit roof area $\left(\mathrm{L} / \mathrm{m}^{2}\right), X$ is water demand, $R$ is reliability, and $P$ is precipitation for a given time period. An example of the SRY curves is shown in Figure 2, where the reliability increases as the storage increases, for a given yield ratio $(\alpha)$. The different values (the power of 2) of storage sizes in Figure 2 were selected to sufficiently cover the domain composed of reliability and yield ratio from 0 to 1 . The yield ratio is defined as a normalized yield (water demand) divided by mean daily rainfall $\left(\mu_{\mathrm{p}}\right)$ of each station per unit area.

$$
\alpha=\frac{X}{\mu_{p}}
$$

For a given $\alpha$, the reliability can be obtained from the number of failure days for given years [19].

$$
R=1-\frac{n_{f}}{n}
$$

where $R$ is reliability, $n$ is the total number of days, and $n_{f}$ is the number of failure days.

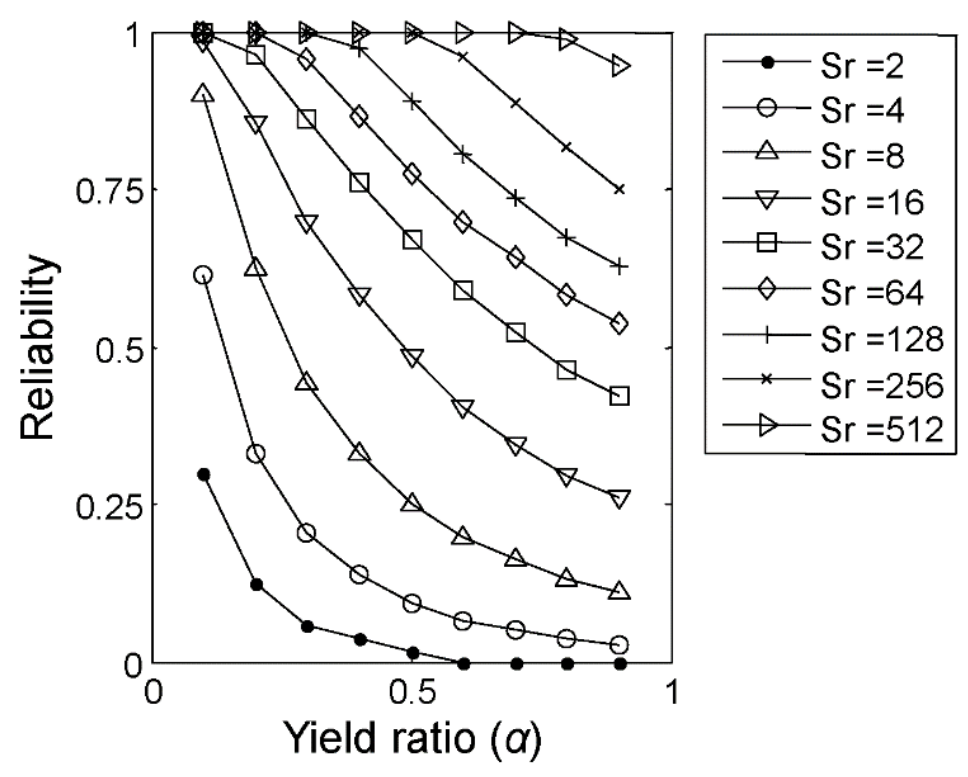

Figure 2. Storage-Reliability-Yield relation curves based on rainfall data in a test catchment, 1018 , depending on yield ratio $(\alpha)$ and storage size $(S r)$ in $\mathrm{L} / \mathrm{m}^{2}$. 
The storage is normalized per unit roof area and, hence, has a dimension of length $\left(\mathrm{L} / \mathrm{m}^{2}\right)$. Assuming that every user possesses a unit area of rainfall catchment, the new set of demands should be normalized with the contributing area before estimating the required storage for the target reliability. Therefore, the total storage of four users can be expressed as follows [23]:

$$
S r_{\text {total }}=\sum S r_{i}=\Sigma\left\{a_{i} \cdot f\left(\frac{C_{i}}{a_{i}}, R, P\right)\right\}
$$

where $S r_{\text {total }}$ is total storage, $S r_{i}$ is the required storage for user $i$, and $C_{i}$ is a new combination of demands created by the sharing network between users of which weight is $a_{i}$, the contributing area obtained by summation of the distributing ratio. For example, let us consider the case (c1) in Table 2, where three users $\left(X_{1}, X_{2}\right.$, and $\left.X_{3}\right)$ are interconnected to each other. In this case, each RWHS is assumed to accommodate every user's water demand equally throughout the RWHS network and the sum of the contributing ratio $\left(a_{1}\right)$ is equal to one. Therefore, the storage size of the RWHS for a user $X_{1}$ is a function of a newly defined demand $C_{1}$ obtained by the SRY relation as follows:

$$
S r_{X_{1}}=f\left(C_{1}, R, P\right)=f\left(\frac{1}{3} X_{1}+\frac{1}{3} X_{2}+\frac{1}{3} X_{3}, R, P\right)
$$

The contributing ratio can be different depending on the type of connection, which can be exclusive to other users and the weight can be greater than one.

\section{Results and Discussion}

\subsection{Storage-Reliability-Yield (SRY) Analysis for a Sharing Network of RWHSs}

The number of connections as shown in Table 2 represents the degree of sharing amongst the RWHSs. Assuming no correlation among users, the required size of total storage is calculated from Equation (10) for each catchment in order to evaluate the effect of establishing sharing networks and the degree of sharing. The result shows that the total required storage of a community can be reduced by sharing storages and the reduction rate increases as the degree of sharing increases. Figure 3 shows the total storage of the community composed of four users as a function of the number of connections (degree of sharing) for four catchments in South Korea. Compared with the total storage without any connections $\left(S_{t}\right)$, total storage $\left(S_{t c}\right)$ was reduced by establishing connections. As shown in Figure 3, the relative total required storage $\left(S_{t 0} / S_{t c}\right)$ is reduced, but the rate of reduction or amount depends on the number of connections, target reliability and location. The result shows that the rate of reduction is smallest in the test catchment, 1001, whereas it is greatest in 5001. As shown in Table 3, the average rainfall duration is bigger in 1001, which is 3.73 days compared with the duration of 2.93 days in 5001. In contrast, the average rainfall intensity is bigger in 5001 compared to that in 1001. It can be inferred that the sharing network of RWHSs are more beneficial in the area, where temporal rainfall variation is greater than others. The result shows that the storage reduction becomes larger as the number of connection $\left(N_{c}\right)$ increases, which is consistent for all test catchments considered in this study. The results also indicate that the rate of reduction increases as the target reliability decreases. For example, Figure $3 \mathrm{~d}$ shows that the required total storage for the community was reduced down to $59 \%$ of the original condition with no RWHS-sharing connections for the target reliability of $85 \%$, whereas the required storage was reduced down to $71 \%$, which is larger, for the target reliability of $95 \%$. 

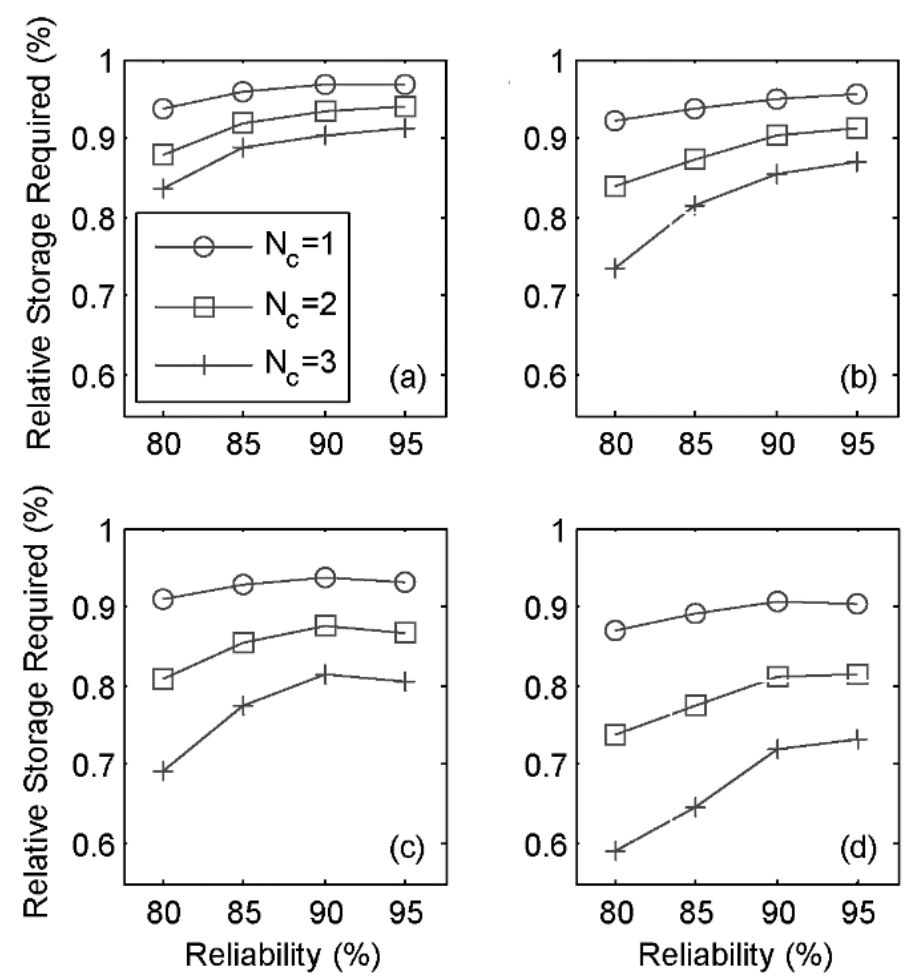

Figure 3. Storage reduction depending on the number of connections $(\mathrm{Nc})$ for target reliabilities of $80 \%, 85 \%, 90 \%$, and 95\% for each catchment: (a) 1001; (b) 1018; (c) 2022; and (d) 5001 with no correlation in demand [DMDV; $\rho=0]$.

The benefit from sharing RWHSs depends not only on the differences in demands among users but also on sharing networks. For example, when two users are sharing RWHSs (the number of connections is one), six possible combinations exist, as shown in Table 2. Figure 4 shows the storage reduction depending on the formation of sharing network when the number of connections is one. Among all possible combinations, the sharing formation of a3 (Table 2) shows the minimum required total storage. This is the case (DMDV) when the user 1 (minimum mean demand) and user 4 (maximum mean demand) share their RWHSs. The result implies that the deficit caused by the largest demand is balanced out by surplus from the smallest demand. This study considers the averages of the results (i.e., total required storage) from all possible combinations hereafter.

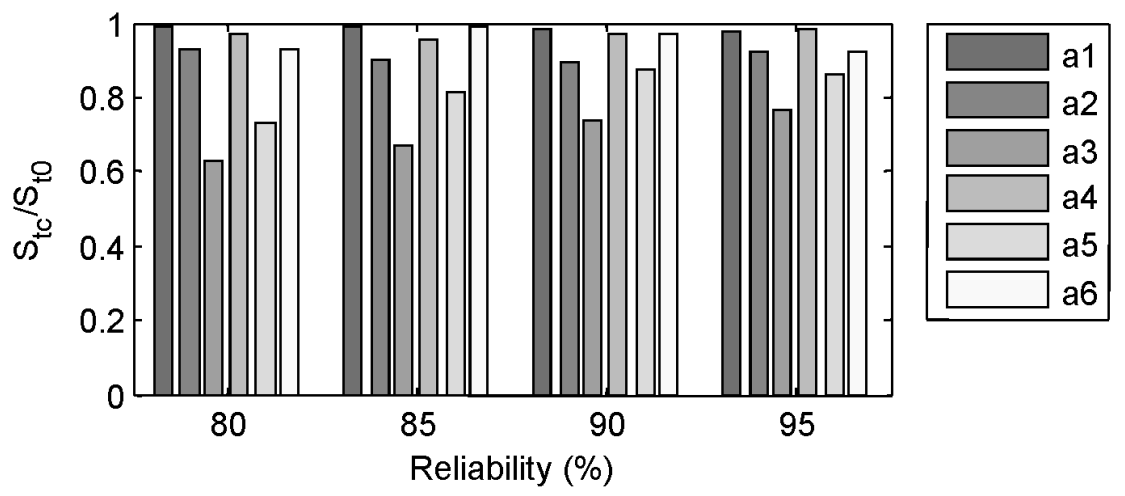

Figure 4. Storage reduction depending on the formation of sharing network when the number of connections is one in a test catchment, 1018 (Seoul), with no correlation in demand. 


\subsection{Demand Characteristics in Water Demand and the Benefit from the Sharing Network}

The correlation in water demand among users is introduced (Figure 5) and the results are compared with the case of no correlation (Figure 3). The results indicate that the correlation in water demand affects the size reduction by sharing RWHSs. As previously shown in Figure 3, the relative total required storage size depending on the number of connections $\left(N_{c}\right)$ compared with the no connection case when the water demands of four users are uncorrelated with each other. In contrast, Figure 5 shows the required total storage with a constant correlation of 0.9 in demand of the users in the community. Figure 3 and Figure 5 both are obtained from the assumption that each demand (user) has different mean and variances). Compared with Figure 3, where no correlation is considered, the results indicate that the effect of correlation on the size reduction depends on the location and target reliabilities. For example, the results shows that the correlation positively affects the sharing benefit (reducing storage size) that the required storage is reduced from $0.72\left(N_{\mathrm{c}}=3\right)$ in Figure $3 \mathrm{~b}$ to 0.67 in Figure $5 \mathrm{~b}$ for the test catchment 1018. However, for the test catchment 2022, the required storage is increased from 0.69 in Figure $3 \mathrm{c}$ to 0.72 in Figure $5 b$, which shows that the correlation negatively affect the sharing benefit. In order to clearly reveal the effect of correlation, the sharing benefit was assessed using a Monte-Carlo simulation by generating correlated demand multiple times.
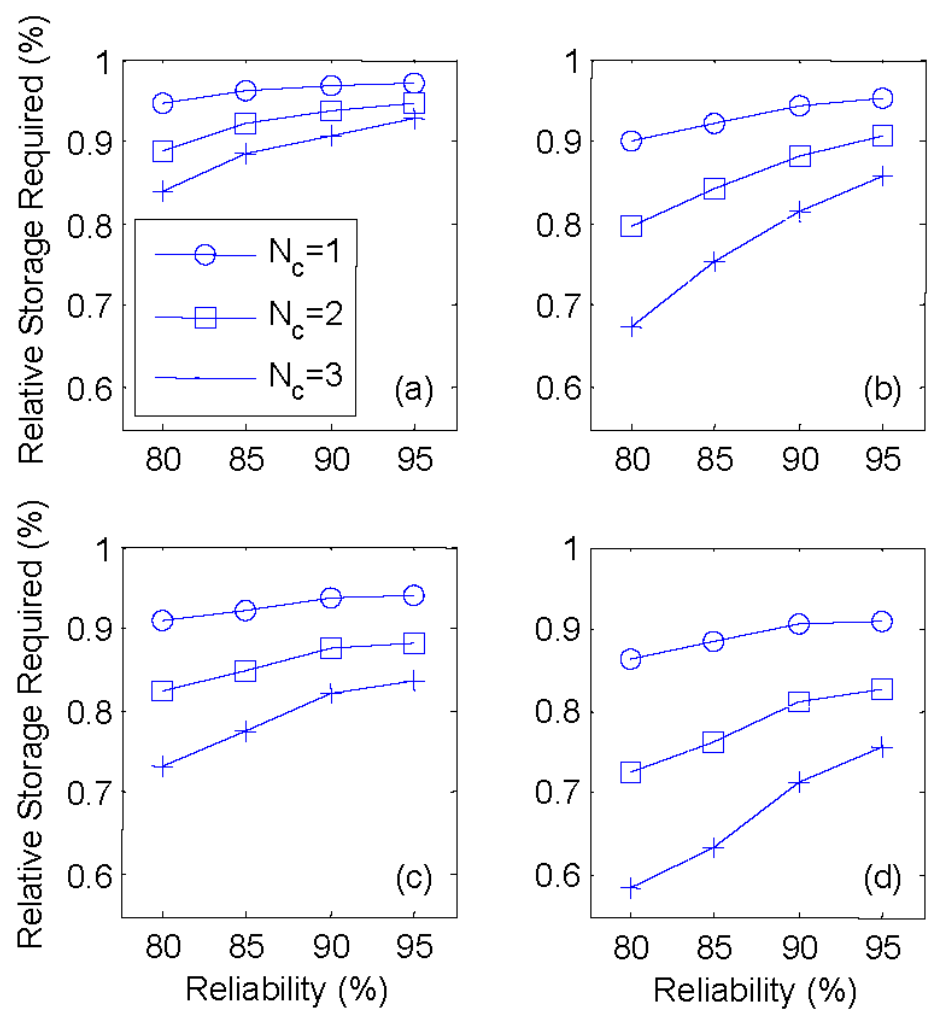

Figure 5. Storage reduction depending on the number of connections $\left(N_{c}\right)$ for target reliabilities of $80 \%, 85 \%, 90 \%$, and 95\% for each catchment: (a) 1001; (b) 1018; (c) 2022, (d) 5001 with correlation in demand [DMDV; $\rho=0.9]$.

In this regard, Figure 6 compares the required total storage when the all users are connected for two cases: one community with a constant correlation (0.9) in demands and the other with no correlation among users in the test catchment, 1018 (Seoul), after one hundred generations of demand series. 
The result clearly reveals that the correlation can enhance the performance of a RWHS whereas uncorrelated demands deteriorate the benefit from a RWHS sharing network. It can be inferred that the performance criteria, reliability only reflects the number of failure of success of the system, but not the severity of the failure at the same time. After one hundred simulations, Table 4 lists the averaged total required storage ratio compared with the unconnected condition for correlated and uncorrelated demands for test catchments. Here, the test case DMDV was considered. As shown in Table 4, the total required storage was reduced when the demands of users are correlated to each other. Table 4 also shows that the correlation in demand positively affects the reliability of a connected RWHSs. For example, for a RWHS sharing network, the total required storage is 0.72 for uncorrelated demands $(\rho=0)$, but it reduces to be 0.69 for correlated demands $(\rho=0.9)$ in the test catchment of 1018 for the target reliability of $80 \%$. As the target reliability decreases, the effect of correlation on the required storage increases as shown in Table 4. For a target reliability of $95 \%$, the required storage does not benefit from the correlation. These results are consistent for all catchments considered in this study.
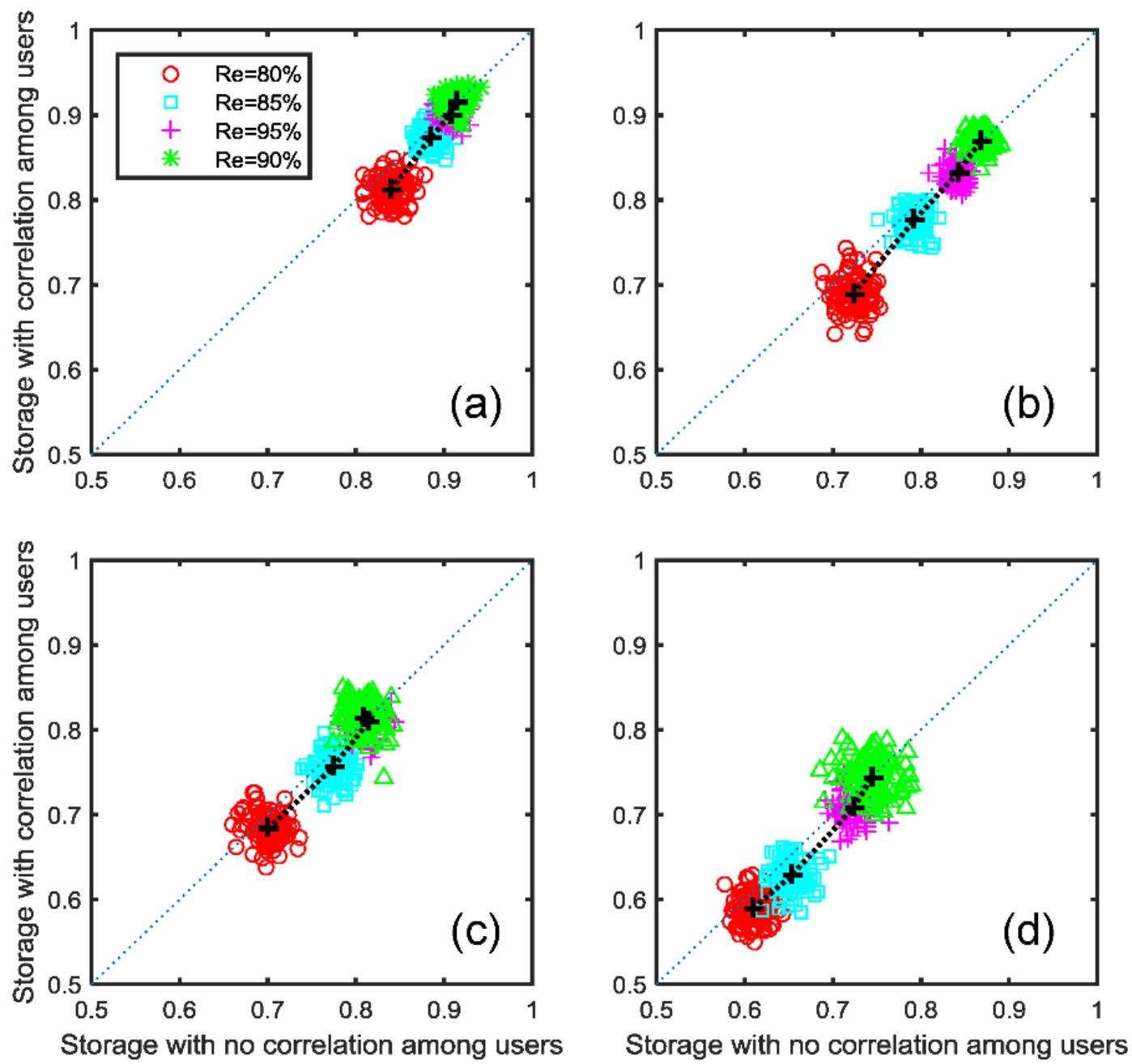

Figure 6. Comparison of the required total storages for correlated and uncorrelated demands when the RWHSs are fully connected for test catchments (a) 1001; (b) 1018; (c) 2022; and (d) 5001 . 
Table 4. Required total storage ratio compared to the unconnected condition for correlated and uncorrelated demands when the RWHSs are fully connected $\left(N_{\mathrm{c}}=3\right)$ for test catchments.

\begin{tabular}{cccccc}
\hline \multirow{2}{*}{ Catchment } & $\boldsymbol{\rho}$ & \multicolumn{4}{c}{ Target Reliability (\%) } \\
\cline { 2 - 6 } & 0 & $\mathbf{8 0}$ & $\mathbf{8 5}$ & $\mathbf{9 0}$ & $\mathbf{9 5}$ \\
\hline \multirow{2}{*}{1001} & 0.9 & 0.84 & 0.89 & 0.91 & 0.91 \\
& 0 & 0.81 & 0.87 & 0.90 & 0.92 \\
\multirow{2}{*}{1018} & 0.9 & 0.72 & 0.79 & 0.84 & 0.87 \\
& 0 & 0.69 & 0.78 & 0.83 & 0.87 \\
\multirow{2}{*}{5022} & 0.9 & 0.70 & 0.77 & 0.81 & 0.81 \\
& 0 & 0.68 & 0.76 & 0.81 & 0.81 \\
& 0.9 & 0.59 & 0.65 & 0.72 & 0.74 \\
& & & 0.63 & 0.71 & 0.74 \\
\hline
\end{tabular}

In order to investigate the effect of demand characteristic more explicitly as shown in Table 1, firstly, the required storage size for each user that satisfies the target reliability of $90 \%$ were estimated. Then, the reliability of the entire community when RWHSs are fully connected was calculated to compare the results with the reliabilities of single users. These results were obtained using demand generated by the Monte-Carlo simulation in the previous section. Figure 7 shows the reliability of four individual users (U1, U2, U3, and U4) in a community when they are no connected and the fully connected case (four users are connected to each other) ( $\mathrm{C}$ in Figure 7) for each test case listed in Table 1. The reliability for individual user in Figure 7 is not exactly 0.9 due to the stochastic nature of demand. Figure 7 compares the reliability of each user and a sharing network for four cases: SMSV, SMDV, DMSV, and DMDV (Table 1). First of all, it should be noted that regardless of differences in mean or variance, the result indicates the positive effect of correlation in demand to the benefit of sharing RWHSs, which is consistent with the previous results. For example, SMDV with $\rho=0$ and 0.9 show that the reliability of the connected system ( $\mathrm{C}$ in Figure 7 ) increases with correlation involved. The same effect of correlation is observed in other cases. The effect of correlation in demand is noticeable when users have different variances in demand (SMDV, DMDV). In contrast, when the users have the same variance, the effect of correlation is smaller.

The comparison of SMSV and DMSV or SMDV and DMDV shows the effect of difference in mean. Figure 7 shows that different means result in greater improvement in system reliability compared with the cases with the same mean, which is consistent whether difference in variance exists or not. Therefore, the result implies that, regardless of variance in demand, sharing RWHSs are more beneficial when users with different means start to share their RWHSs with each other. In addition, here in this case, it should be noted that the increase in reliability, when RWHSs are connected, is independent of variance. On the other hand, the comparison of SMSV and SMDV or DMSV and DMDV reveals the effect of difference in variance. When the mean demand is equal to each other, different variance in demand leads to lower reliability when RWHSs are connected; this is consistent when the mean demand is different to each other. Therefore, the result implies differences in variance give rise to negative impact on the resulting reliability when a sharing network is introduced. In addition, it should be noted that decrease in reliability, when RWHSs are connected, is independent of mean. To summarize the results, DMSV produces the largest increase in reliability by introducing a sharing network and correlation has a positive impact on the reliability of the connected RWHSs. 

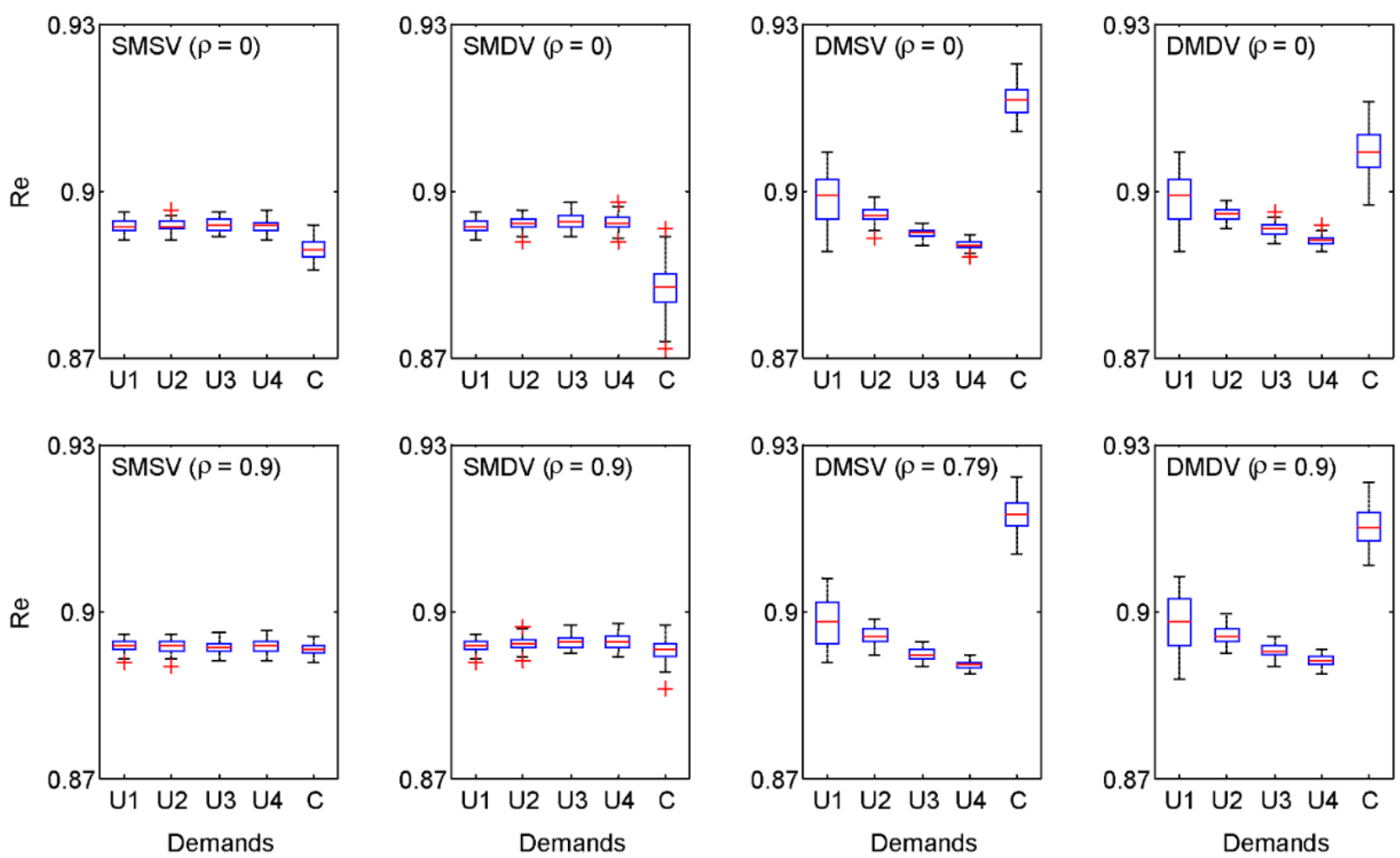

Figure 7. Reliability of four users (U1, U2, U3, and U4) in a community and the fully connected case $(\mathrm{C})$ for each test case (1018).

The resiliency can be written as follows [32],

$$
\gamma=\frac{1-R}{r_{f}}
$$

where $\gamma$ is resiliency, $R$ is reliability and $r_{f}$ is the ratio of total number of days when the condition moves from success to failure divided by the total number of days in a year. Figure 8 shows the resiliency of four individual users (U1, U2, U3, and U4) in a community and the fully connected case (C in Figure 8) for each test case listed in Table 1. Figure 8 compares the resiliency of each user and a sharing network for four cases: SMSV, SMDV, DMSV, and DMDV. In terms of resiliency, the results show opposite results compared with reliability. First of all, it should be noted that the result shows the little effect of correlation in demand on the resiliency of the connected RWHS system. The comparison of SMSV and DMSV or SMDV and DMDV shows the effect of difference in mean. The resiliency of the connected system was decreased when the mean demands are different to each other, but it was not decreased nor increased with the same mean; this is consistent when variances are different. The decrease in resiliency remains constant whether the variances are same or not. The storage size for each user was obtained with the target reliability of $90 \%$. However, Figure 8 shows that the resiliency shows different behavior from reliability. The resiliency increases as the variance increases (SMDV) whereas it decreases as the mean increases (DMSV). In addition, DMDV shows that the difference in mean dominates the effect of different variances in demand in terms of resiliency. 

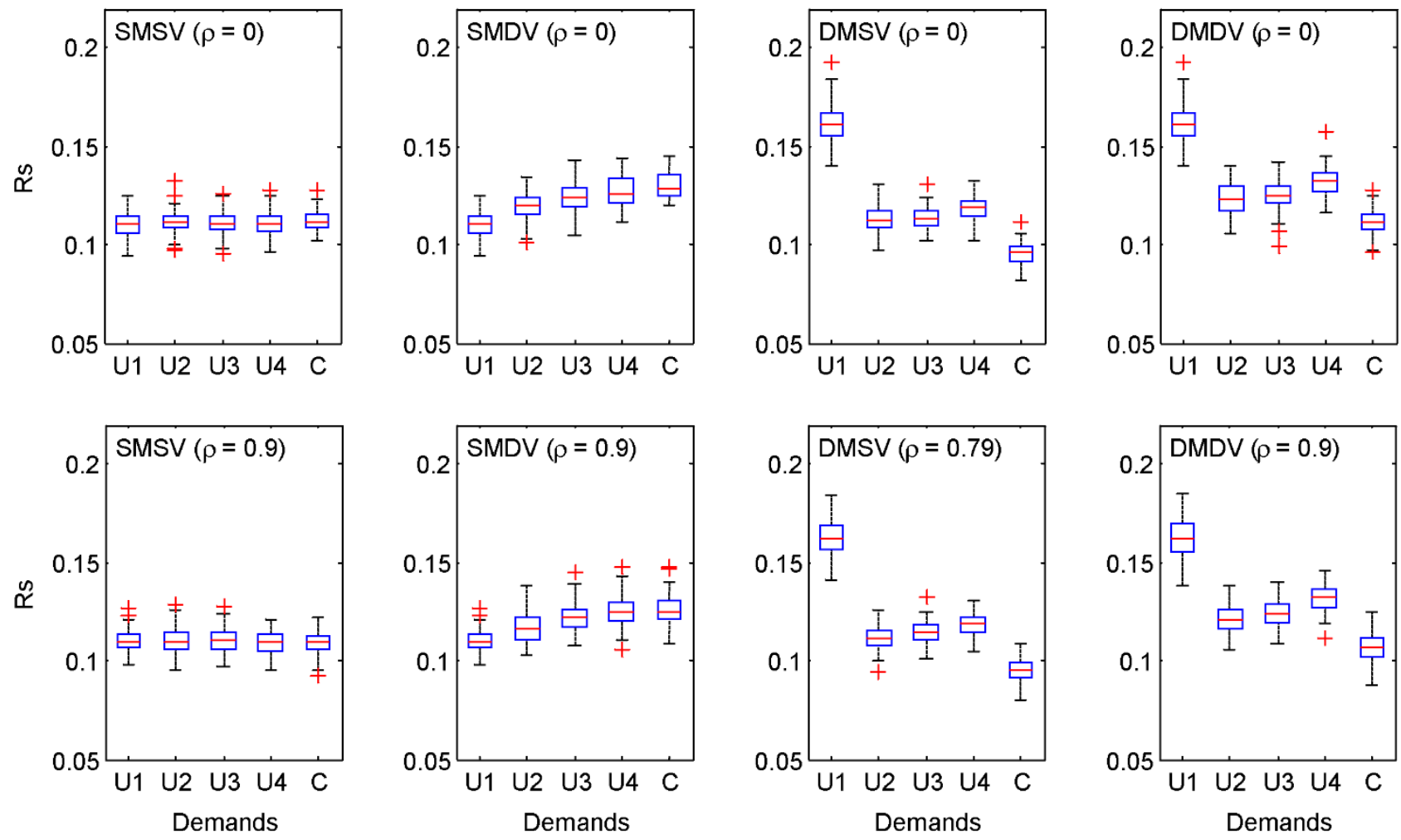

Figure 8. Resiliency of four users (U1, U2, U3, and U4) in a community and the fully connected RWHSs (C) for each test case (1018).

On the other hand, the comparison of SMSV and SMDV or DMSV and DMDV reveals the effect of difference in variance on the resiliency of the connected system. The result shows that the resiliency of the connected system was increased with difference variances. In addition, these results are consistent with the case of different means (DMSV, DMDV). Therefore, it can be inferred that difference variances lead to increase in resiliency of the connected RWHS system. In the same way, the increase in resiliency with different variance remains constant regardless of the mean demand. In summary, DMSV shows the lowest resiliency of the connected system whether correlation in demand exists or not. In general, correlation has little impact on resiliency of the connected system. However, the impact of correlation in demand should be more explicitly investigated in terms of severity, which would be examined in the future study.

Figures 6 and 7 showed the reliability and resiliency of the connected system for the test catchment, 1018 only. Figure 9 shows the averaged reliability and resiliency of the all test catchment considered in this study. The results show that the behavior of the performance criteria in the test catchment 1018 is consistent with other three catchments, 1001, 2022, and 5001 in South Korea. The cases of constant mean in Figure 9 (SMSV, SMDV) consistently show that the reliability was negatively affected by sharing RWHSs in the community. However, the resiliency is positively affected by the sharing RWHSs for the constant mean cases (SMSV, SMDV). In contrast, the results for DMSV and DMDV show that the difference in mean increases reliability but decreases resiliency when sharing RWHSs is introduced. SMDV also shows that the reliability decreases even more when the variances of demands are different to each other. In contrast, the cases of varying mean in Figure 9 consistently result in increased reliability of the entire system when all the RWHSs are shared. In addition, the result from DMSV implies the 
negative impact of different variances of demands, which is opposite to the case of SMSV. Overall, both the averaged reliability and resiliency are not affected by correlation in demand.

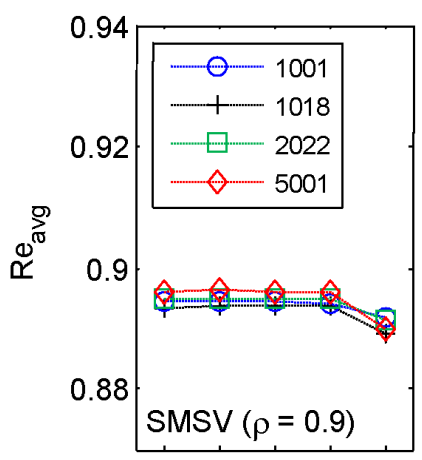

U1 U2 U3 U4 $\mathrm{C}$

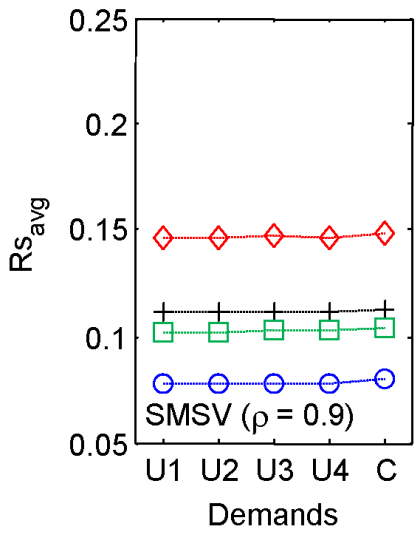

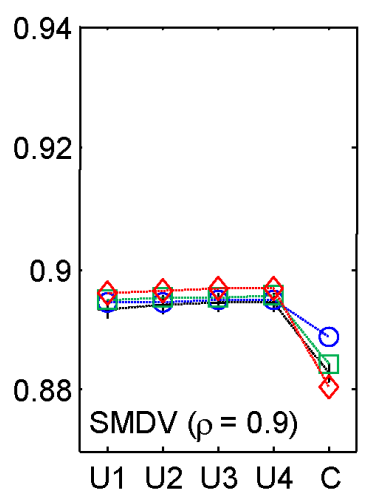

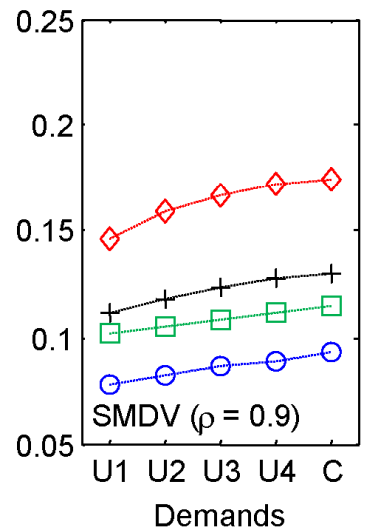

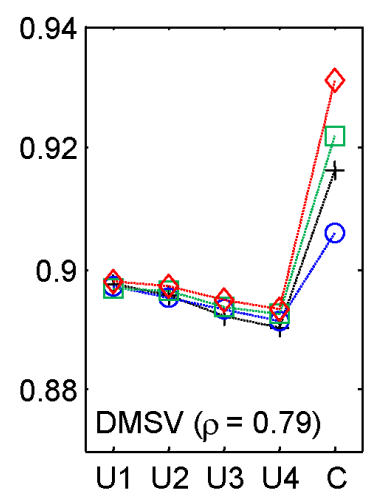
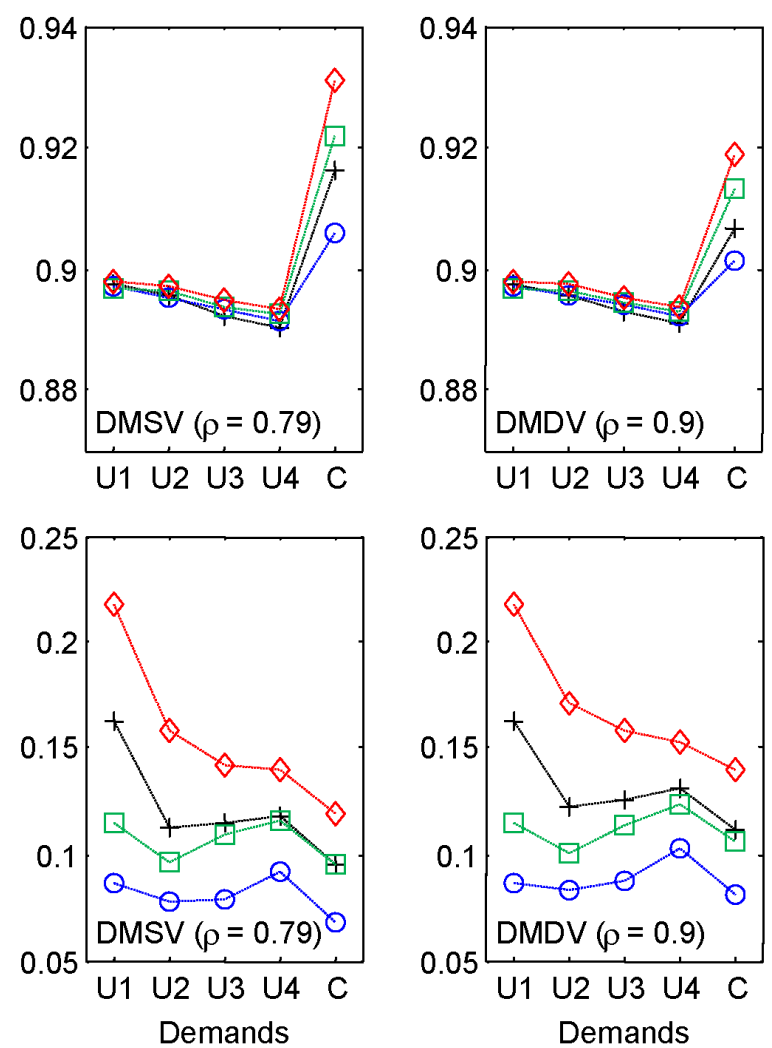

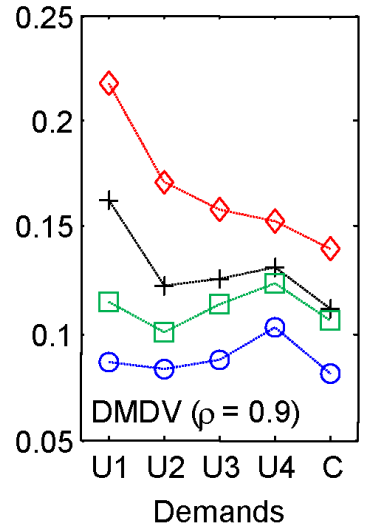

Figure 9. Averaged reliability and resiliency of four users (U1, U2, U3, and U4) in a community and the fully connected RWHSs (C) for all catchments.

\section{Conclusions}

This study investigated the benefit from a network of sharing RWHSs introducing different characteristics in water demand. Individual could potentially benefit from sharing, which is more feasible in urban areas. This study examines the possibility of reducing total required storages by sharing RWHSs utilizing historical daily rainfall in four catchments in South Korea. Test cases with different means, variances, and correlation in water demand among four prospective users in a sample community are introduced and its impact on the benefit derived from a sharing network of RWHSs is examined. The results indicate that the degree of sharing is closely related with the performance of the RWHS; the total required storage of a community can be reduced by sharing storages up to $39 \%$ and the reduction rate increases as the degree of sharing increases. Therefore, highly shared RWHSs improve the reliability of the system. The results showed that correlation in water demand among users additionally reduces the required storage up to $3 \%$ and, hence, it contributes to reliability of the entire system, which were consistent for all test catchments in South Korea. Test cases with different means and variances as well as correlation disclose the conditions when the benefit from sharing RWHSs is most expected. The results imply the maximum potential benefit from a RWHS sharing network with different means and variances in terms of reliability and resiliency. The benefit was observed in all test catchments considered in this study. The results also showed that reliability may not sufficient to evaluate the performance of a RWHS. Vulnerability concept can be additionally included to assess the severity 
of failures of a RWHS. Improving a network of RWHSs in terms of severity of failure using a RWHS sharing network should be addressed but left for future studies.

Overall, the benefit from sharing is heavily dependent on the different characteristics in water demand among users and the formation of the sharing network, which suggests an optimal sharing network maximizing the reliability of a system. Therefore, the results of this study imply the potential improvement of RWHSs through establishing an optimum network for sharing individual storages. Consequently, this paper demonstrates the benefits and limitations of RWHS connections, which will improve the understanding of the behavior of connected RWHSs and give insights to communities and public decision makers to improve RWHS performances.

\section{Acknowledgments}

This study was supported by Basic Science Research Program by the National Research Foundation of Korea (NRF) from the Ministry of Science, ICT and Future Planning (NRF-2013R1A1A1058964).

\section{Author Contributions}

Yongwon Seo designed the numerical experiment. Young-Oh Kim collected the rainfall data for future climate change scenarios. Yongwon Seo and Sun Young Park conducted the analysis of the performance of RWHS networks. The manuscript was written by Yongwon Seo with contribution from Sun Young Park, and Young-Oh Kim.

\section{Conflicts of Interest}

The authors declare no conflict of interest.

\section{References}

1. Pacey, A.; Cullis, A. Rainwater Harvesting: The Collection of Rainfall and Runoff in Rural Areas; ITDG Publishing: London, UK, 1986.

2. Ludwig, A. Water Storage: Tanks, Cisterns, Aquifers, and Ponds for Domestic Supply, Fire and Emergency Use; Oasis Design: Santa Barbara, CA, USA, 2005.

3. Su, M.-D.; Lin, C.-H.; Chang, L.-F.; Kang, J.-L.; Lin, M.-C. A probabilistic approach to rainwater harvesting systems design and evaluation. Resour. Conserv. Recycl. 2009, 53, 393-399.

4. Abdulla, F.A.; Al-Shareef, A.W. Roof rainwater harvesting systems for household water supply in Jordan. Desalination 2009, 243, 195-207.

5. Fewkes, A. Modelling the performance of rainwater collection systems: Towards a generalized approach. Urban Water 2000, 1, 323-333.

6. Ghisi, E.; Bressan, D.L.; Martini, M. Rainwater tank capacity and potential for potable water savings by using rainwater in the residential sector of southeastern Brazil. Build Environ. 2007, 42, 1654-1666.

7. Imteaz, M.A.; Rahman, A.; Ahsan, A. Reliability analysis of rainwater tanks: A comparison between South-East and Central Melbourne. Resour. Conserv. Recycl. 2012, 66, 1-7. 
8. Basinger, M.; Montalto, F.; Lall, U. A rainwater harvesting system reliability model based on nonparametric stochastic rainfall generator. J. Hydrol. 2010, 392, 105-118.

9. Villarreal, E.L.; Dixon, A. Analysis of a rainwater collection system for domestic water supply in Ringdansen, Norrköping, Sweden. Build Environ. 2005, 40, 1174-1184.

10. Fox, P.; Rockström, J.; Barron, J. Risk analysis and economic viability of water harvesting for supplemental irrigation in semi-arid Burkina Faso and Kenya. Agric. Syst. 2005, 83, 231-250.

11. Liaw, C.H.; Tsai, Y.L. Optimum storage volume of rooftop rain water harvesting systems for domestic use. J. Am. Water Resour. Assoc. 2004, 40, 901-912.

12. Daigger, G.T. Evolving urban water and residuals management paradigms: Water reclamation and reuse, decentralization, and resource recovery. Water Environ. Res. 2009, 81, 809-823.

13. Jenkins, D.; Pearson, F.; Moore, E.; Sun, J.K.; Valentine, R. Feasibility of Rainwater Collection SYstem in California; California Water Resources Center, University of California: Davis, CA, USA, 1978.

14. Mitchell, V.G. How important is the selection of computational analysis method to the accuracy of rainwater tank behaviour modelling? Hydrol. Process. 2007, 21, 2850-2861.

15. Guo, Y.P.; Baetz, B.W. Sizing of rainwater storage units for green building applications. J. Hydrol. Eng. 2007, 12, 197-205.

16. Howard, C. Theory of storage and treatment-plant overflows. J. Environ. Eng. Div. 1976, 102, $709-722$.

17. Loganathan, G.V.; Delleur, J.W. Effects of urbanization on frequencies of overflows and pollutant loadings from storm-sewer overflows-A derived distribution approach. Water Resour. Res. 1984, 20, 857-865.

18. Adams, B.; Papa, F. Urban Stormwater Management Planning with Analytical Probabilistic Models; John Wiley \& Sons Inc: New York, NY, USA, 2000.

19. Hanson, L.; Vogel, R.; Kirshen, P.; Shanahan, P.; Starrett, S. Generalized Storage-Reliability-Yield Equations Rainwater Harvesting Systems. In Proceedings of the World Environmental \& Water Resources Congress 2009, Kansas City, MO, USA, 2009; pp. 1-10.

20. Kuria, F.; Vogel, R. Global storage-reliability-yield relationships for water supply reservoirs. Water Resour. Manag. 2014, 29, 1591-1605.

21. Imteaz, M.A.; Ahsan, A.; Naser, J.; Rahman, A. Reliability analysis of rainwater tanks in melbourne using daily water balance model. Resour. Conserv. Recycl. 2011, 56, 80-86.

22. Rahman, A.; Keane, J.; Imteaz, M.A. Rainwater harvesting in Greater Sydney: Water savings, reliability and economic benefits. Resour. Conserv. Recycl. 2012, 61, 16-21.

23. Seo, Y.; Choi, N.J.; Park, D. Effect of connecting rain barrels on the storage size reduction. Hydrol. Process. 2012, 26, 3538-3551.

24. Seo, Y.; Ahn, J.; Kim, Y.-O. The impact of climate change on the benefit of a rain barrel sharing network. Resour. Conserv. Recycl. 2013, 74, 66-74.

25. Young, R.A. Price elasticity of demand for municipal water: A case study of Tucson, Arizona. Water Resour. Res. 1973, 9, 1068-1072.

26. Maidment, D.; Parzen, E. Time patterns of water use in six Texas cities. J. Water Resour. Plan. Manag. 1984, 110, 90-106. 
27. Balling, R.C.; Gober, P. Climate variability and residential water use in the city of Phoenix, Arizona. J. Appl. Meteorol. Climatol. 2007, 46, 1130-1137.

28. Rahman, M.; Yusuf, F.U.A.M.S. Rainwater harvesting and the reliability concept. In Proceedings of the 8th ASCE Specialty Conference on Probabilistic Mechanics and Structural Reliability, Washington, DC, USA, 2000.

29. Surendran, S.; Tanyimboh, T.; Tabesh, M. Peaking demand factor-based reliability analysis of water distribution systems. Adv. Eng. Softw. 2005, 36, 789-796.

30. Xu, C.; Goulter, I. Reliability-based optimal design of water distribution networks. J. Water Resour. Plan. Manag. 1999, 125, 352-362.

31. Hajani, E.; Rahman, A. Reliability and cost analysis of a rainwater harvesting system in peri-urban regions of Greater Sydney, Australia. Water 2014, 6, 945-960.

32. Hashimoto, T.; Stedinger, J.R.; Loucks, D.P. Reliability, resiliency, and vulnerability criteria for water resource system performance evaluation. Water Resour. Res. 1982, 18, 14-20.

(C) 2015 by the authors; licensee MDPI, Basel, Switzerland. This article is an open access article distributed under the terms and conditions of the Creative Commons Attribution license (http://creativecommons.org/licenses/by/4.0/). 